\title{
Effects of Water Contamination on the Supercooled Dynamics of a Hydrogen-Bonded Model Glass Former
}

\author{
Johan Sjöström, ${ }^{*}$ Rikard Bergman, Carl Wadell, Tobias Moberg, and Jan Swenson \\ Department of Applied Physics, Chalmers University of Technology, SE-41296 Göteborg, Sweden
}

Johan Mattsson

School of Physics \& Astronomy, University of Leeds, Leeds, LS2 9JT, U.K.

ABSTRACT: Broad-band dielectric spectroscopy is a commonly used tool in the study of glass-forming liquids. The high sensitivity of the technique together with the wide range of probed time scales makes it a powerful method for investigating the relaxation spectra of liquids. One particularly important class of glass-forming liquids that is often studied using this technique consists of liquids dominated by hydrogen $(\mathrm{H})$ bond interactions. When investigating such liquids, particular caution has to be taken during sample preparation due to their often highly hygroscopic nature. Water can easily be absorbed from the atmosphere, and dielectric spectroscopy is a very sensitive probe of such contamination due to the large dipole moment of water. Our knowledge concerning the effects of small quantities of water on the dielectric properties of these commonly investigated liquids is limited. We here demonstrate the effects due to the presence of small amounts of water on the dielectric response of a typical H-bonded model glass former, tripropylene glycol. We show how the relaxation processes present in the pure liquid are affected by addition of water, and we find that a characteristic water induced relaxation response is observed for water contents as low as 0.15 wt $\%$. We stress the importance of careful purification of hygroscopic liquids before experiments and quantify what the effects are if such procedures are not undertaken.

\section{INTRODUCTION}

Broad-band dielectric spectroscopy is a useful and commonly applied tool in the study of relaxation dynamics of supercooled liquids. ${ }^{1}$ Many organic liquids can be supercooled within an experimentally easily accessible temperature range, and their supercooled dynamics are of importance for many technological, biological, and medical applications. ${ }^{2}$ Molecules with strong inherent dipole moments are particularly suitable for dielectric investigations. Thus, liquids such as glycerol or tripropylene glycol (3PG) are often used as model glass-forming liquids. ${ }^{1,3}$ However, the polar nature of such liquids often makes them hygroscopic and particular care has to be taken during sample preparation in order to avoid misleading results arising from contamination by water. It has previously been demonstrated that water can clearly affect the spectra of 3PG and other propylene glycol oligomers, ${ }^{4,5}$ and careful drying of these liquids gives rise to spectra that differ significantly from spectra of the liquids used "as is". 5,6

Many natural polymers, such as proteins or polysaccharides, also display a high degree of $\mathrm{H}$-bonding and thus uptake water naturally from the atmosphere. Hydration can affect the different relaxation modes of biopolymers, and there are various reports in the literature regarding how high the hydration level needs to be to cause a substantial effect on the dynamics. ${ }^{7,8}$ As one example, the $\alpha$ relaxation of cellulose speeds up whereas the side group dynamics slows down ${ }^{9,10}$ if water is present on the percentage level.

Strongly H-bonded liquids are often water soluble, which can be utilized when investigating the interplay between water and organic solutes or when attempting to study the properties of supercooled water, which in its pure form inevitably crystallizes below $238 \mathrm{~K}$ due to homogeneous nucleation. A number of studies show the existence of a water induced relaxation process in mixtures of water and other molecules. ${ }^{11-16}$ This relaxation process is generally faster than the structural relaxation and has commonly been described as a relaxation process of relatively local character, ${ }^{11,14,16,17}$ resembling the intrinsic secondary $\beta$ relaxation, which is a generic property of pure glass formers. ${ }^{18-20}$ The behavior of this water induced relaxation is best characterized in the glassy

Received: August 25, 2010

Revised: January 7, 2011

Published: February 7, 2011 
state of mixtures containing more than 20 wt \% water, where it carries several typical characteristics: (i) its dielectric strength increases systematically with increasing water content, (ii) it exhibits an Arrhenius temperature dependence with an activation energy of $\sim 0.5 \mathrm{eV}$, (iii) the dielectric loss is symmetric on a logarithmic frequency scale, (iv) no calorimetric glass transition can be easily identified as the relaxation time crosses the experimental time scale, and (v) the relaxation becomes faster with increasing water content, such that its relaxation time, $\tau$, for a fixed temperature in the glassy state obeys $\tau \sim \exp \left(-a C_{\mathrm{w}}\right)$, where $C_{\mathrm{w}}$ is the water mass fraction. ${ }^{16}$ Interestingly, a relaxation process with very similar properties appears also in studies of pure water subjected to "hard" confinement including clays, ${ }^{21}$ zeolites, ${ }^{22}$ or porous silica. $^{23-25}$ These similarities strongly support the interpretation of the observed water induced relaxation feature as mainly due to the relaxation of water molecules. However, for low water contents $(<10 \mathrm{wt} \%)$ the water induced relaxation does not decouple from the structural relaxation within the experimentally accessible time scales $(\sim 100 \mathrm{~s})$.

We here perform a systematic investigation of the effects that small amounts of water have on the supercooled and glassy dynamics of $3 \mathrm{PG}$, which is a commonly used model glassforming liquid for which $\mathrm{H}$-bonding interactions play an important role. The dielectric loss spectrum of pure 3PG exhibits both a structural $\alpha$ relaxation and a secondary $\beta$ relaxation. ${ }^{2,5,6,26-31}$ We study the relaxation dynamics of both freeze-dried pure 3PG and 3PG to which controlled amounts of water are added in order to quantify the effects of water addition. A water induced relaxation process is detected for water contents as low as $0.15 \mathrm{wt} \%$. It is also demonstrated how the $\alpha$ and $\beta$ relaxations of the pure liquid are affected for these low water contents. The study clearly confirms the importance of careful purification of hygroscopic liquids in order to avoid spurious contributions due to water uptake.

\section{EXPERIMENTAL SECTION}

3PG was purchased from Sigma-Aldrich (CAS Registry No. 24800-44-0; product no. 187593-1L; purity $>97 \%$ ) and purified using the following protocol: The liquid was contained in a round-bottom flask that was connected to a turbo pump via a cold trap held at $78 \mathrm{~K}$. The liquid was depressurized to less than $10^{-5}$ mbar and left until all appearing bubbles had disappeared. The liquid was then vitrified by lowering the flask into liquid nitrogen, where it was left for $5 \mathrm{~min}$, after which it was reheated by use of a heating gun. Additional bubbles were observed as the system devitrified. The cooling/reheating routine was repeated 13 times, after which no bubbles were observed upon reheating. ${ }^{32}$ This procedure has previously been used to remove water impurities from $\mathrm{H}$-bonded molecular liquids and polymer melts. ${ }^{6,16,16,33-35}$ To verify the proceedure, we performed a Karl Fischer titration on $3 \mathrm{PG}$ that was subjected to the freeze-drying protocol described above. With a sample size of $1.1 \mathrm{~g}$ and a coulometric titration cell from Metrohm (Titrando 852), the water content in freeze-dried 3PG was determined to be less than 0.004 wt $\%$ ( $\pm 3 \mathrm{ppm})$. The freeze-dried 3PG served as a reference sample and as a starting material for the mixtures. Deionized water of conductivity less than $0.05 \mu \mathrm{S} / \mathrm{cm}$ was added to the dry 3PG in controlled amounts, and the mixtures, kept in tight containers, were sonicated in an ultrasonic water bath for $15 \mathrm{~min}$ to ensure good mixing. Samples of $100 \mu \mathrm{m}$ thickness (the thickness was controlled by the use of three silica spacers) were sandwiched between brass electrodes with a diameter of $20 \mathrm{~mm}$

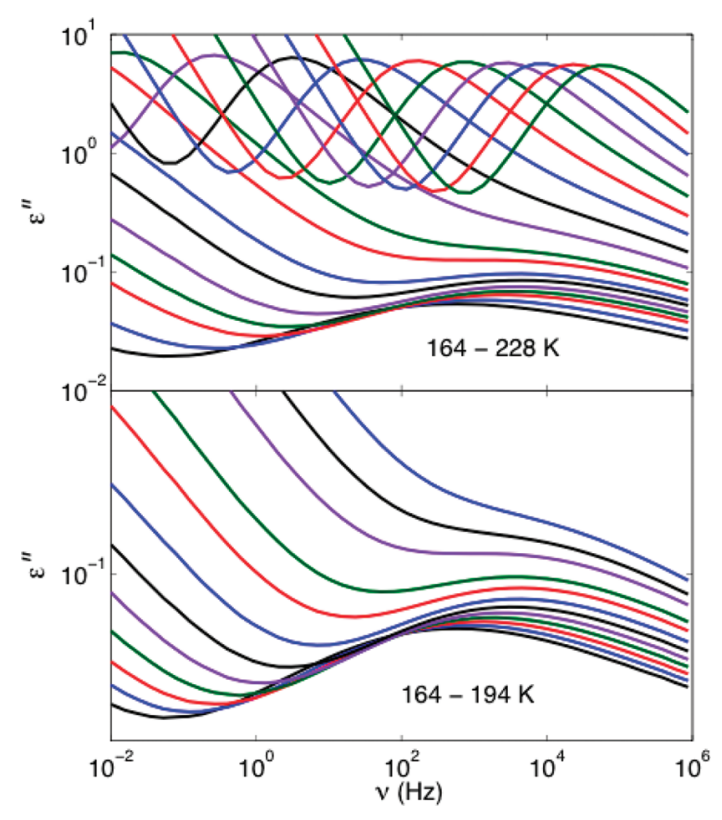

Figure 1. Dielectric loss spectra of anhydrous, freeze-dried 3PG. Upper panel: $164-185 \mathrm{~K}$ in steps of $3 \mathrm{~K}$ and 187,190 , and $192-228 \mathrm{~K}$ in steps of $4 \mathrm{~K}$. Lower panel: rescaled to highlight the $\beta$ relaxation region and also includes $194 \mathrm{~K}$.

and placed within a sealed sample cell. The dielectric loss was measured in a frequency range of $10 \mathrm{mHz}-1 \mathrm{MHz}$ upon cooling in 38 steps, from 259 to $115 \mathrm{~K}$, using broad-band dielectric spectroscopy (Novocontrol Alpha).

\section{RESULTS AND DISCUSSION}

Dielectric loss spectra of pure freeze-dried 3PG are shown in Figure 1 for temperatures ranging from the glassy to the supercooled state. The strength of the dielectric loss for the secondary $\beta$ relaxation is about a factor 100 less compared with that of the structural $\alpha$ relaxation. Both relaxations become faster with increasing temperatures and they are well separated up to temperatures just above the glass transition, $T_{\mathrm{g}}=190 \mathrm{~K}$, defined as $T_{\mathrm{g}}=T\left(\tau_{\alpha}=100 \mathrm{~s}\right)$; at higher temperatures the two relaxations merge. The results for pure $3 \mathrm{PG}$ as well as $3 \mathrm{PG}$ to which water was added are shown in Figure 2 for concentrations ranging from 0.25 to $30 \mathrm{wt} \%$ at $T=175 \mathrm{~K}$. For this temperature all samples are in their glassy state and the rise toward low frequencies is due to the high-frequency flank of the $\alpha$ relaxation loss peak situated at frequencies outside the experimental window. The loss peak of the secondary $\beta$ relaxation is clearly resolved at $\sim 10^{3}-10^{4} \mathrm{~Hz}$, as shown in Figure 2.

As water is added, however, the strength of the $\beta$-loss peak is reduced and a new loss process instead appears at $\sim 10^{-1}-10^{1} \mathrm{~Hz}$. This has previously been observed for water concentrations above 4 wt \%. ${ }^{4}$ As shown in Figure 2, however, this relaxation process is visible even at subpercentage water contents, although it does not give rise to a clear peak for such low water contents since it is partly hidden by the high-frequency flank of the structural $\alpha$ relaxation. With increasing water concentrations the strength of this relaxation clearly grows, and for large water contents it dominates the spectrum, as demonstrated in Figure 2. This relaxation contribution clearly corresponds to the water induced relaxation commonly observed for a wide range of materials. ${ }^{12-16}$ 


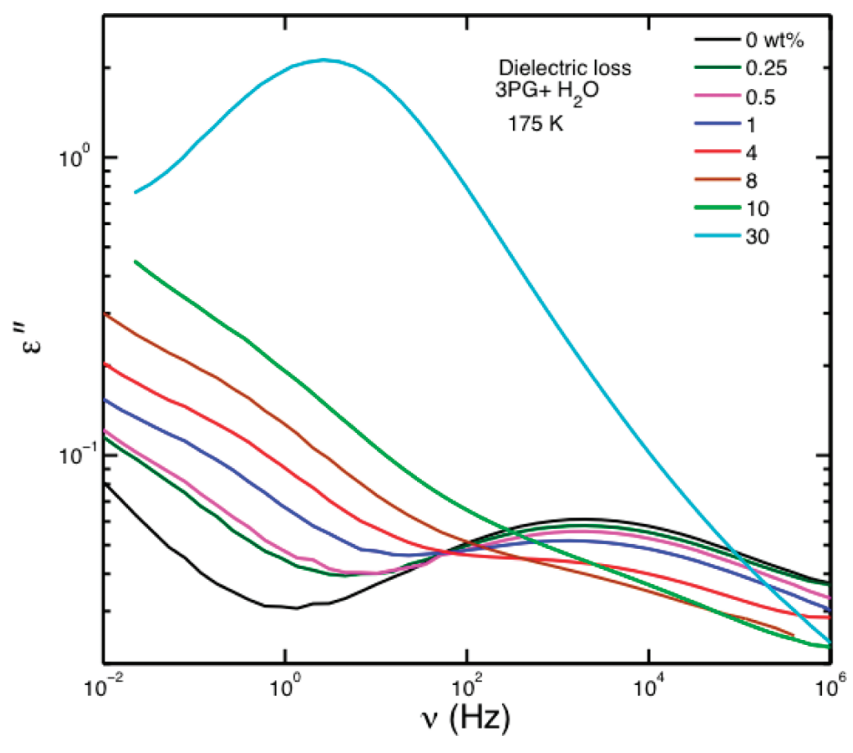

Figure 2. Dielectric loss spectra of freeze-dried 3PG and 3PG with controlled amounts of added water, respectively, at $T=175 \mathrm{~K}$. Note that all samples are in their glassy state at this temperature.

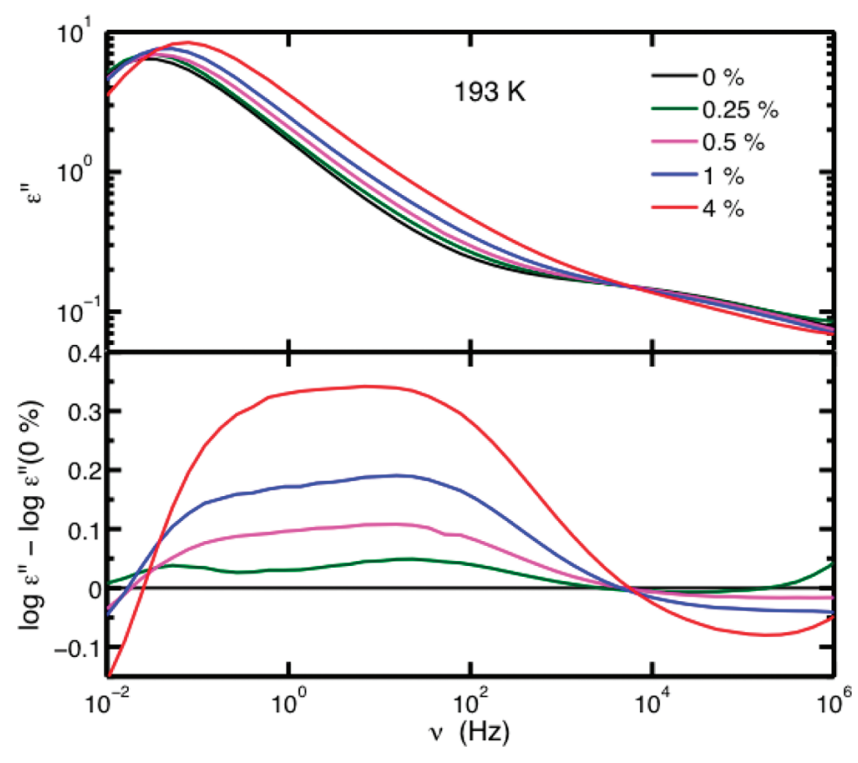

Figure 3. Upper panel: dielectric loss spectra of 3PG/water mixtures at $193 \mathrm{~K}$. Lower panel: the difference in logarithmic dielectric loss between the mixtures and the freeze-dried pure 3PG sample.

For temperatures just above the glass transition temperature $\left(T_{g}=190 \mathrm{~K}\right)$, the structural relaxation loss peak enters the experimental window at low frequencies, which makes the water induced intermediary relaxation process less marked. Figure 3 (upper panel) shows the dielectric loss spectra at $193 \mathrm{~K}$ for water contents between 0 and $4 \%$; the spectra contain the loss peaks corresponding to the structural $\alpha$ relaxation, the intrinsic secondary $\beta$ relaxation, and the water induced relaxation. We find that even samples with $\leq 1$ wt $\%$ water exhibit shifts in position and intensity of their $\alpha$ relaxation. To better resolve the differences between the anhydrous 3PG and the 3PG-water mixtures, we subtract the anhydrous spectra from the other spectra, resulting in the residual spectra shown in the lower panel of Figure 3. We clearly see that the difference spectra can be

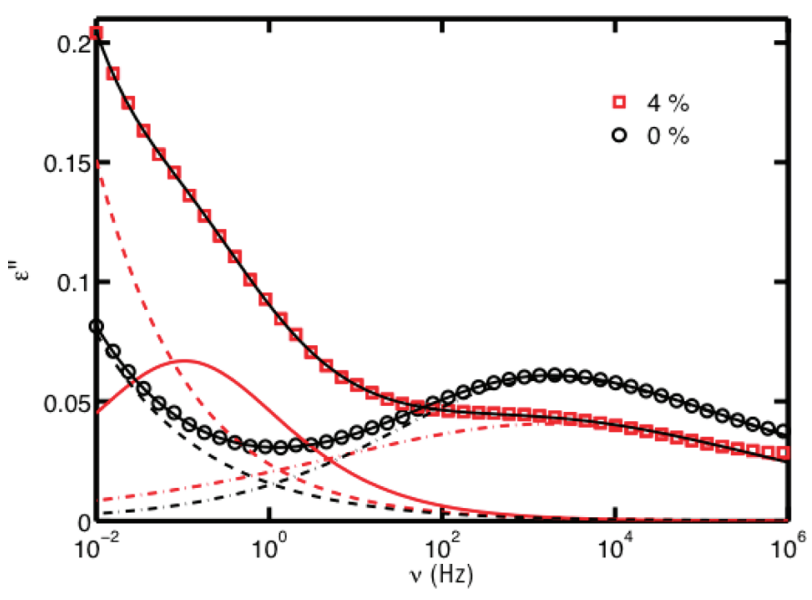

Figure 4. Examples of fitting results at $T=175 \mathrm{~K}$ for freeze-dried $3 \mathrm{PG}$ and $3 P G$ with 4 wt $\%$ added water. The dashed-dotted lines are the CC functions describing the intrinsic $\beta$ relaxation. The dashed lines are power laws describing the $\alpha$ relaxation tail (with the same exponent), and the red solid line is the contribution from the water induced process in the sample with $4 \mathrm{wt} \%$ water. The black solid lines are the total resulting fits.

divided into three parts. The increase in the relative dielectric loss at low frequencies is partly due to an increase in the strength of the $\alpha$ relaxation, which is partly related to the increased dipole moment density as water is added, and partly due to a slight shift of the $\alpha$ relaxation toward higher frequencies. The latter plasticization effect has previously been thoroughly investigated for higher water contents. ${ }^{11,12,16}$ The contribution to the difference spectra at $\sim 10^{0}-10^{3} \mathrm{~Hz}$ originates from the water induced relaxation. Clearly, this contribution is present also in the supercooled regime for water contents as low as 0.25 wt $\%$. The (negative) contribution to the difference spectra at high frequencies is due to the decrease in intensity of the $\beta$ relaxation as water is added. ${ }^{4}$

In order to quantify the effects of water addition, we fit the spectra at $175 \mathrm{~K}$ using Cole-Cole (CC) functions, ${ }^{36} \varepsilon_{\mathrm{CC}}^{\prime \prime}=-$ $\operatorname{Im}\left\{\Delta \varepsilon /\left[1+(\mathrm{i} \omega \tau)^{a}\right]\right\}$, for both the water induced relaxation and the secondary $\beta$ relaxation. We account for the tail of the $\alpha$ relaxation, which affects the spectra at low frequencies, by using a power-law expression, $\varepsilon_{\alpha}^{\prime \prime}=A / \omega^{\delta}$. An example of the resulting fits is shown in Figure 4. For high water contents we keep all parameters free, but for low water contents, the power-law exponent of the $\alpha$ relaxation tail is fixed to the value for the anhydrous sample, $\delta=0.4$, although the amplitude remains a free parameter. The two methods do not produce any systematic difference for the dielectric strength of the water induced relaxation as seen in Figure 5.

The dielectric strengths determined from the fits of the water induced relaxation are shown in Figure 5. We find that the strength of the water induced relaxation process varies systematically with water content from the lowest $(0.15 \mathrm{wt} \%)$ to the highest (30 wt \%) measured water fractions, and as the water content approaches zero the strength becomes negligible and eventually vanishes, as confirmed by the lack of evidence for this relaxation process for the purified dry $3 \mathrm{PG}$.

To quantify the spectra at $T>T_{g}$, we fit the data with a sum of standard fitting functions, including a Havriliak-Negami (HN) function for the structural relation ${ }^{37}$ and two CC functions describing the water induced relaxation (with the dielectric strength fixed to the values shown in Figure 5) and the intrinsic 


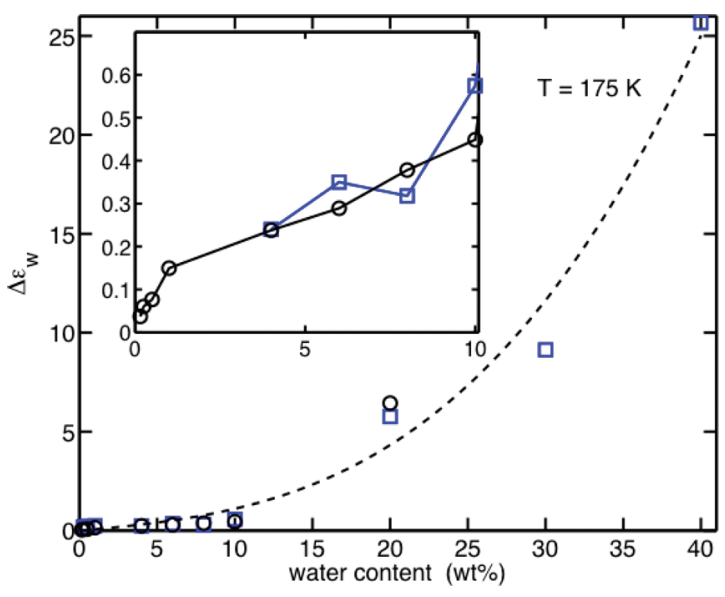

Figure 5. Dielectric strength of the water-related process at $175 \mathrm{~K}$. The circles result from the fitting procedure with the contribution from the $\alpha$ relaxation described by a power law with the exponent set to the value obtained for the anhydrous sample. The squares result from fits with all parameters free. The dashed line is a guide to the eye. The inset shows the same data for low water concentrations.

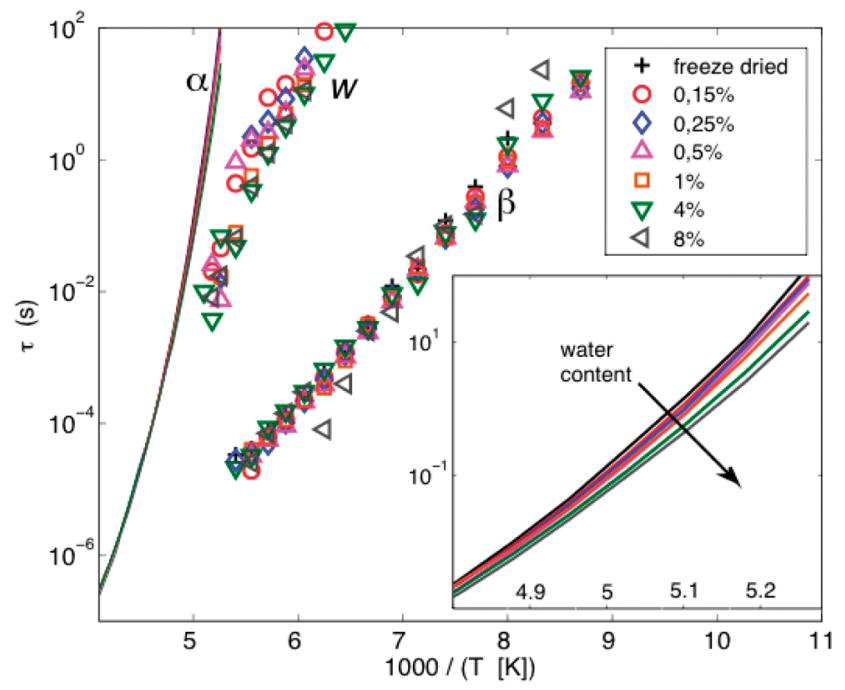

Figure 6. Arrhenius plot for the $\alpha, \beta$, and water induced relaxation $(w)$. The inset shows the $\alpha$ relaxation in the temperature range near the glass transition.

secondary $\beta$ relaxation, respectively. The conductivity contribution is fit with a $\sigma_{0} / \varepsilon_{0} \omega$ term, where $\sigma_{0}$ is the dc conductivity and $\varepsilon_{0}$ is the vacuum permittivity. We find that the structural $\alpha$ relaxation becomes systematically faster with increasing water content, as can be seen from the relaxation times shown in Figure 6. This is in agreement with previous findings at higher water contents, ${ }^{4,16}$ and as a consequence $T_{\mathrm{g}}$ decreases by $1 \mathrm{~K}$ for a hydration level of $1 \%$; see Figure 7.

The water induced relaxation also becomes faster with increasing water content, consistent with the behavior observed for higher water contents. ${ }^{16}$ We do not find any significant shift of the secondary $\beta$ relaxation. Such shifts have been previously observed for secondary relaxations related to side group motions in hydrated cellulose. ${ }^{9,10}$ We observe an almost linear increase in conductivity with increasing water fraction. Moreover, we find that the strength of the $\alpha$ relaxation increases approximately

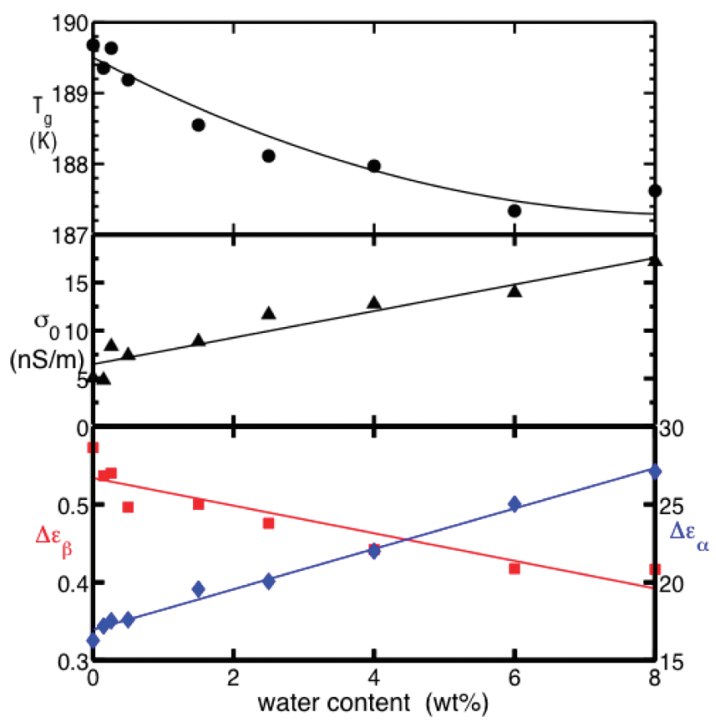

Figure 7. Fitting results for different water contents. The glass transition temperature (circles), the dc conductivity (triangles), and the dielectric strength of the $\alpha$ (diamonds) and intrinsic $\beta$ relaxation (squares) are shown. The lines are guides to the eye.

linearly with water content, whereas the strength of the intrinsic $\beta$ relaxation decreases upon addition of water. The behavior of the $\alpha$ relaxation strength can be understood based on the cooperative nature of the structural relaxation together with the highly polar nature of the added water. Interestingly, the opposite effect is found for the more local $\beta$ relaxation.

To further illustrate the importance of sample purification, we measured the dielectric loss for a sample of 3PG obtained directly from a previously unopened sample container purchased from Sigma-Aldrich (CAS Registry No. 24800-44-0; product no. $187593-25 \mathrm{ML})$. The purity for this specific batch was confirmed by the supplier to be $>99 \mathrm{wt} \%$. Consistent with this, we determind its water content to 0.43 wt $\%$ by Karl Fischer titration. The bottle was opened in a humidity-free atmosphere within an $\mathrm{Ar}$ glovebox, and the sample cell was prepared within the box. Thereafter, the cell was transferred within a sealed plastic bag to the cryostat in which nitrogen gas was flowing in order to prevent any uptake of water from the air. 3PG from the same bottle was subsequently freeze-dried according to the protocol described above and remeasured. The loss spectra obtained for the two different preparation procedures differ significantly, as is evident from Figure 8 . We observe differences in the behavior of the $\alpha$ and the $\beta$ relaxations as well as in the dc conductivity. Moreover, we clearly find evidence for a water induced relaxation for the non-freeze-dried sample, as seen in the $T=175 \mathrm{~K}$ data in Figure 8. The dielectric loss of the "as obtained" sample matches the one measured for a mixture with a water concentration of $0.5-1$ wt $\%$ water, which is consistent with the degree of purity given by the manufacturer. Thus, the "as obtained" liquid might be appropriate for studies of qualitative features, but for detailed analysis of the spectra, especially in the glassy state or for a quantitative determination of conductivity, further purification is clearly needed.

Interestingly, in several studies a weak secondary relaxation feature, corresponding to the water induced relaxation studied in this work, has been interpreted as an intrinsic so-called JohariGoldstein $\beta$ relaxation of the pure material. ${ }^{27-31}$ For 3PG, studies have been performed at both ambient and elevated pressures. The 


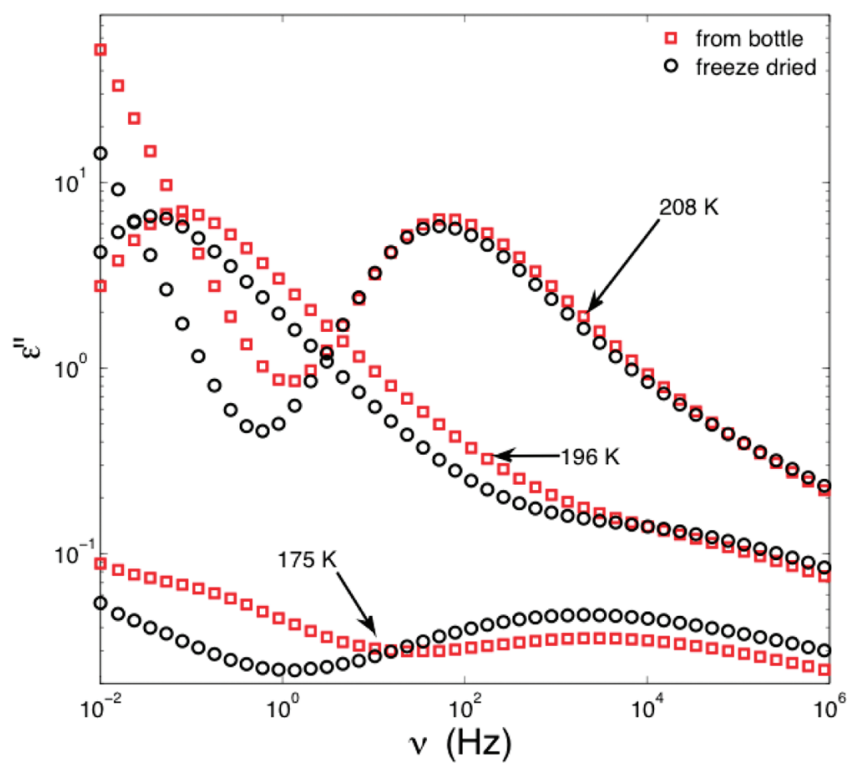

Figure 8. Dielectric loss spectra at three different temperatures for 3PG measured as obtained from Sigma-Aldrich (red squares) and from the same bottle after careful freeze-drying of the sample (black circles).

relaxation was most clearly resolved at elevated pressures, but was also observed at ambient conditions as an excess wing on the high-frequency flank of the $\alpha$ relaxation. ${ }^{27-31}$ For a longer chain, oligo(propylene glycol), a relaxation peak ${ }^{31}$ was observed in the same dynamic range. In light of our findings, one expects such effects if water is present even on a subpercentage level, and since we do not observe this relaxation for the dry sample, it appears that the secondary relaxation observed in refs $27-30$ is due to water contamination of the samples.

One could argue that small amounts of water present in the sample could act as a probe of a weak intrinsic dielectric relaxation that is otherwise not observable. The question of how to distinguish a relaxation induced by water from an intrinsic, not directly observable, dielectric relaxation which slaves water motion is not easy to resolve. However, the systematic variation of the relaxation strength over the full water concentration range shown in Figure 5, the approach toward zero strength for small water contents, and the lack of a relaxation for a carefully dried sample strongly suggest that the observed relaxation in these studies $^{27-30}$ is caused by water present in the sample. Since our measurements were performed at ambient pressures only, we cannot exclude that an additional relaxation might be visible at higher pressures since, when increasing the pressure, the $\alpha$ relaxation is shifted toward lower frequencies and a relaxation present in between the $\alpha$ and the intrinsic $\beta$ relaxation might be better resolved. ${ }^{29}$

\section{- CONCLUSIONS}

We have studied the dielectric response of the $\mathrm{H}$-bonded liquid tripropylene glycol, 3PG, both in its dry state and upon addition of various amounts of water. We demonstrate that the effect of water on the dielectric loss spectrum is clearly evident already for concentrations well under $1 \mathrm{wt} \%$. A water induced relaxation, which is commonly observed in the dielectric relaxation of water-containing systems, is observed in mixtures with water contents as low as $0.15 \mathrm{wt} \%$. We find that the dielectric strength of this relaxation systematically approaches zero for decreasing water content, and for the pure 3PG sample we find no evidence for the extra relaxation. Moreover, we observe an identical relaxation feature also for 3PG taken directly from a freshly opened sample container obtained from the manufacturer (Sigma-Aldrich, with specified purity $>99 \%$ ). Careful freeze drying of this sample, however, removes the loss contribution, demonstrating the importance of careful sample preparation procedures in investigations of hygroscopic liquids such as 3PG.

\section{AUTHOR INFORMATION}

\section{Corresponding Author}

*E-mail: johan.sjostrom@chalmers.se.

\section{ACKNOWLEDGMENT}

This work was financially supported by the Swedish Research Council and the Swedish Energy Agency.

\section{REFERENCES}

(1) Schneider, U.; Brand, R.; Lunkenheimer, P.; Loidl, A. Phys. Rev. Lett. 2000, 84, 5560.

(2) Pethig, R.; Kell, D. B. Phys. Med. Biol. 1987, 32, 933.

(3) Leon, C.; Ngai, K. L.; Roland, C. M. J. Chem. Phys. 1999, 110, 11585 .

(4) Grzybowska, K.; Grzybowski, A.; Pawlus, S.; Hensel-Bielowka, S.; Paluch, M. J. Chem. Phys. 2005, 123, 204506.

(5) Gainaru, C.; Hiller, W.; Böhmer, R. Macromolecules 2010, 43, 1907.

(6) Mattsson, J.; Bergman, R.; Jacobsson, P.; Börjesson, L. Phys. Rev. B 2009, 79, 174205.

(7) Einfeldt, J.; Maissner, D.; Kwasniewski, A. Prog. Polym. Sci. 2001, 26, 1419.

(8) Sousa, M.; Brás, A. R.; Veiga, H. I. M.; Ferreira, F. C.; de Pinho, M. N.; Correia, N. T.; Dionísio, M. J. Phys. Chem. B 2010, 114, 10939. 6977.

(10) Jafarpour, G.; Roig, F.; Dantras, E.; Boudet, A.; Lacabanne, C. J. Non-Cryst. Solids 2009, 355, 1669.

(11) Cerveny, S.; Schwartz, G.; Bergman, R.; Swenson, J. Phys. Rev. Lett. 2004, 93, 245702.

(12) Cerveny, S.; Schwartz, G.; Alegria, A.; Bergman, R.; Swenson, J. J. Chem. Phys. 2006, 124, 194501.

(13) Shinyashiki, N.; Sudo, S.; Yagihara, S.; Spanoudaki, A.; Kyritsis, A.; Pissis, P. J. Phys.: Condens. Matter 2007, 19, 205113.

(14) Capaccioli, S.; Ngai, K. L.; Shinyashiki, N. J. Phys. Chem. B 2007, $111,8197$.

(15) Cerveny, S.; Alegria, A.; Colmenero, J. Phys. Rev. E 2008, 77, 031803.

(16) Sjöström, J.; Mattsson, J.; Bergman, R.; Johansson, E.; Josefsson, K.; Svantesson, D.; Swenson, J. Phys. Chem. Chem. Phys. 2010, 12, 10452.

(17) Swenson, J.; Jansson, H.; Bergman, R. Phys. Rev. Lett. 2006, 96, 247806.

(18) Johari, G. P.; Goldstein, M. J. Chem. Phys. 1970, 53, 2372.

(19) Ngai, K. L.; Paluch, M. J. Chem. Phys. 2004, 120, 857.

(20) Mattsson, J.; Bergman, R.; Jacobsson, P.; Börjesson, L. Phys. Rev. Lett. 2003, 90, 075702.

(21) (a) Bergman, R.; Swenson, J. Nature (London) 2000, 403, 283. (b) Bergman, R.; Swenson, J.; Börjesson, L.; Jacobsson, P. J. Chem. Phys. 2000, 113, 357.

(22) Jansson, H.; Swenson, J. Eur. Phys. J. E 2003, 12, s51.

(23) Pissis, P.; Laudat, J.; Daoukaki, D.; Kyritsis, A. J. Non-Cryst. Solids 1994, 171, 201.

(24) Spanoudaki, A.; Albela, B.; Bonneviot, L.; Peyrard, M. Eur. Phys. J. E 2005, 17, 21. 
(25) (a) Hedström, J.; Swenson, J.; Bergman, R.; Jansson, H.; Kittaka, S. Eur. Phys. J. Spec. Top. 2007, 141, 53. (b) Sjöström, J.; Swenson, J.; Bergman, R.; Kittaka, S. J. Chem. Phys. 2008, 128, 154503.

(26) Dyre, J. C.; Olsen, N. B. Phys. Rev. Lett. 2003, 91, 155703.

(27) Casalini, R.; Roland, C. M. Phys. Rev. Lett. 2003, 91, 015702.

(28) Casalini, R; Roland, C. M. Phys. Rev. B 2004, 69, 094202.

(29) Roland, C. M.; Hensel-Bielowka, S.; Paluch, M.; Casalini, R. Rep. Prog. Phys. 2005, 68, 1405.

(30) Pawlus, S.; S. Hensel-Bielowka, S.; Paluch, M.; Casalini, R.; Roland, C. M. Phys. Rev. B 2005, 72, 064201.

(31) Grzybowska, K.; Grzybowski, A.; Zioło, J.; Paluch, M.; Capaccioli, S. J. Chem. Phys. 2006, 125, 044904.

(32) For liquids of low vapor pressure it is often sufficient to leave them under vacuum $\left(10^{-5} \mathrm{mbar}\right)$ for a long time, $\sim 24 \mathrm{~h}$ at a temperature far above $T_{\mathrm{g}}$, while using a liquid nitrogen cooled cold trap. Liquids with higher vapor pressure will however be sucked into the trap or pump under such conditions, and freeze-thaw cycling should be used.

(33) Bergman, R.; Börjesson, L.; Torell, L. M.; Fontana, A. Phys. Rev. B 1997, 56, 11619.

(34) Carlsson, P.; Swenson, J.; Börjesson, L.; Torell, L. M.; McGreevy, R. L.; Howells, W. S. J. Chem. Phys. 1998, 109, 8719.

(35) Mattsson, J.; Bergman, R.; Jacobsson, P.; Börjesson, L. Phys. Rev. Lett. 2005, 94, 165701.

(36) Cole, K. S.; Cole, R. H. J. Chem. Phys. 1941, 9, 341.

(37) Havriliak, S.; Negami, S. Polymer 1967, 8, 161. 